into the sea bed, the ability to become cemented to hard substrates, or the ability to escape by swimming (scallops), was generally followed by a rapid proliferation of the group.

J.D. Taylor (British Museum (Natural History)) similarly showed how the success of neogastropods ( 20,000 species) can be traced back to their radiation in the Cretaceous, some 100 million years ago, when they began to evolve a fiendish array of predatory adaptations. Some can bore holes into hard-bodied prey through which they suck their victims' flesh; others can catch prey with a long snake-like proboscis; some swallow their prey whole by powerful suction; others have complex venom glands and even modified hollow 'teeth' that are shot into their prey like hypodermic poison darts. Unlike many of these predatory adaptations, hole-boring predation can be tracked back in the fossil record. Fossil shells with holes that were bored by particular gastropods became more common during the Cretaceous. This occurred in parallel with the radiation of the hole-borers, thus providing cogent evidence for the radiation and effectiveness of at least this adaptation.

Other contributors found less evidence that key adaptations could explain the radiations of their chosen groups of organisms. M.B. Hart (Plymouth Polytechnic) emphasized the role of repeated extinction events and opportunistic radiation in the fossil record of planktonic foraminifera. After an extinction event, the survivors, which seem to have been mainly surface-living forms, radiated to occupy deeper positions in the water column. Extinctions over the past 250 million years have also triggered radiations of echinoderms (M.J. Simms, Trinity College, Dublin) and terrestrial tetrapods (A.R. Milner, Birkbeck College, London; my own work). By contrast, evolutionary radiations of colonial animals involved major structural changes in the colony form that are best explained by heterochronic change. Evidence was presented for the corals (B.R. Rosen, British Museum and J.M. Pandolfi, Australian Institute of Marine Science), bryozoans (P.D. Taylor, British Museum; G.P. Larwood, University of Durham), and for the extinct, largely planktonic colonial graptolites (C.E. Mitchell, State University of New York, Buffalo).

Several contributors addressed the socalled null or neutral model, which states that species may diversify by chance alone, and not for any particular biological or physical reason. Although the issue was considered in biological and statistical terms, the consensus was very much against this model.

Radiations at higher levels than the individual adaptive radiation may also yield general patterns and models of evolutionary interest. The diversification of the tetrapods, for example, is largely the result of the conquest of new 'adaptive space' and the subdivision of niches, or increasing specialization of species and families. There does not seem to have been any in-built progressive 'tendency' in their evolution. D. Jablonski (University of Chicago) and D.J. Bottjer (University of Southern California, Los Angeles) note that marine invertebrates have repeatedly shown environmental patterns of radiation. Most of the main groups arose in shallow waters and then migrated over tens of millions of years into deeper seas. New groups then replaced them in the near-shore waters. This remarkable 'conveyor belt' is unexpected and may have parallels elsewhere. Skelton and coauthors find evidence that most of the main bivalve groups arose at high latitudes and subsequently migrated towards equatorial seas; Jablonski and Bottjer, however, find that most other marine groups arose at low latitudes and migrated north and south. Similarly, P.R. Crane and S. Lidgard (Field Museum of Natural History, Chicago) report an equatorial origin for flowering plants, with a subsequent migration to temperate latitudes.

A potential problem with studies of evolutionary radiations is the incompleteness of the fossil record. This may be a serious problem in studies of the well known Early Cambrian radiation, some 570 million years ago, when hard parts first appeared in the fossil record, and molluscs, arthropods, echinoderms and others apparently radiated very rapidly. It is not known whether this seemingly rapid radiation is preceded by a significant gap in the fossil record ( $\mathrm{S}$. Conway Morris, University of Cambridge). Most parts of the fossil record are, however, complete enough for valid macroevolutionary studies, with many hundreds of fossil deposits showing exceptional preservation. These have provided information on soft-bodied organisms, as well as hardbodied organisms with delicate structures Together, they enable palaeontologists to interpret with confidence those intervening parts of the fossil record showing poorer preservation.

The other key problem in studying radiations is establishing the precise geometry of such patterns, the shape of the tree of the history of life. Most of the 21 studies presented at the meeting were based on new cladistic analyses, a rigorous technique for assessing patterns of lineage splitting. Cladistic techniques are becoming the norm for macroevolutionary studies and promise increasing success for the palaeobiological study of evolutionary radiations.

Michael J. Benton is in the Department of Geology, University of Bristol, Wills Memorial Buildings, Queen's Road, Bristol BS8 1RJ, UK

\section{Floating on air}

THE hovercraft supports itself on a cushion of pressurized air, maintained between its underside and the ground. But lacking sides, the cushion leaks absurdly freely. Air has to be pumped down into it at an enormous rate to sustain the pressure. Daedalus is now designing a novel leak-proof hovercraft. It pumps air inwards into its cushion from all round its periphery, exactly opposing the outward leakage.

The basic principle is that oxygen, and therefore air, is paramagnetic. So it can be entrained and pumped by a moving magnetic field. The periphery of the DREADCO hovercraft is studded with linear magnetic motors, whose field pattern runs radially inwards at high speed. These motors pump air in under the craft until sufficient pressure has built up to oppose further inward flow. Acting on weakly paramagnetic air, even the most powerful linear motors can only pressurize the central cushion by a tiny fraction of an atmosphere - but over the whole bottom area of the craft this should be enough to float several tons.

This novel vehicle has two great advantages. First, once established, its supporting air cushion will be quite static. With no fans or air draughts, the magnetic hovercraft will be utterly silent. It will float above the ground as enigmatically as a flying saucer. Second, the magnetic pumps merely serve to maintain a static pressure-gradient across the peripheral air of the cushion. They do no work of compression and with luck will consume very little energy. The craft should be very efficient.

As with all hovercraft, propulsion poses a problem. Bleeding off cushion air for jet propulsion would sabotage its lift; biasing the linear motors to drive the craft along a metallic track would tie it down like a train to a railway. Daedalus hopes to build a novel jet engine on the same principle as his magnetic air-pump: a long tubular linear motor, sucking air in at one end and blowing it out the other. Even modest thrust from such a quiet and simple engine should give his frictionless vehicle a useful turn of speed. And two parallel engines could be biased against each other to steer it.

The magnetic hovercraft should be very popular with environmentalists and nature lovers. Alone among heavier-than-air vehicles, it will produce no downwash. It will travel quietly over crops and meadows and swamps and tidelands, without alarming the wild life or flattening the vegetation. In towns, however, its stray field may be something of a liability. It will be greatly perturbed by iron manhole covers and tram tracks, and may ingest beer cans and scrap metal. Daedalus plans to give the craft magnetic sensors which momentarily switch off any linear motor that encounters such embarrassing challenges. David Jones 